Supporting information for the paper:

\title{
Investigations of the antibacterial properties of ciprofloxacin@ $\mathrm{SiO}_{2}$
}

\author{
M.J. Rosemary, ${ }^{1}$ lan MacLaren ${ }^{2 \dagger}$ and T. Pradeep ${ }^{1 *}$ \\ 1DST Unit on Nanoscience, Department of Chemistry and Sophisticated Analytical Instrument \\ Facility, Indian Institute of Technology, Madras, Chennai 600 036, India. \\ 2 Department of Physics and Astronomy, University of Glasgow, Glasgow G12 8QQ, United \\ Kingdom.
}

*For correspondence, Email: pradeep@iitm.ac.in, Fax: 00-91-44-2257-0509 or 0545

$\dagger$ At the Institute for Materials Science, Darmstadt University of Technology, Petersenstr. 23, 64287 Darmstadt, Germany while this work was initiated. 


\section{Supporting information 1}

\section{Method of synthesis of silica nanoshells}

Gold nanoparticles of size $15 \mathrm{~nm}$ were prepared using the Turkevich reduction method. To $200 \mathrm{~mL}$ of the gold sol, $1 \mathrm{~mL}$ of millimolar of ciprofloxacin was added under vigorous stirring and the solution was allowed to stand for 15 minutes so that complete complexation of ciprofloxacin on gold surface took place. Next, $1.5 \mathrm{~mL} 1 \mathrm{mM}$ solution of freshly prepared APS was added to it with vigorous stirring. This mixture was again allowed to stand for around 15 minutes for complete complexation. A solution of active silica was prepared by adjusting the $\mathrm{pH}$ to $10-11$ of a $0.54 \mathrm{wt} \%$ of sodium silicate solution by progressive addition of a cation exchange resin, Dualite C $225-\mathrm{Na}$ 14- 52 mesh. $10 \mathrm{~mL}$ of active silica thus prepared was added to $200 \mathrm{~mL}$ of the surface modified gold sol. The resulting mixture was allowed to stand for one day so that the active silica polymerizes on the surface of the gold particle to form $\mathrm{Au} @ \mathrm{SiO}_{2}$.

$\mathrm{Au} @ \mathrm{SiO}_{2}$ thus obtained was centrifuged for around one hour and the particles were collected and were repeatedly washed with 2-propanol to make sure that no ciprofloxacin was present on the surface of silica. This material was re-dispersed in a mixture of 2-propanol and water in the ratio, 4:1. To this, $2 \mathrm{M}$ sodium cyanide solution was added to remove the gold core and stirred for around 48 hours. The reaction between the gold core and sodium cyanide was monitored by UV/VIS spectroscopy. The dissolution of gold was confirmed by the disappearance of the gold plasmon.

Cip@ $\mathrm{SiO}_{2}$ was centrifuged out and washed several times with water to ensure that there was no ciprofloxacin or ions adhering to the outer surface of silica. This was confirmed by the fact that the centrifugate, after removing cip@SiO ${ }_{2}$, did not show any fluorescence/absorbance features of ciprofloxacin/Au3 ${ }^{3+}$. Redispersed cip@SiO 2 showed the ciprofloxacin features (Figure S2). 


\section{Supporting information 2}

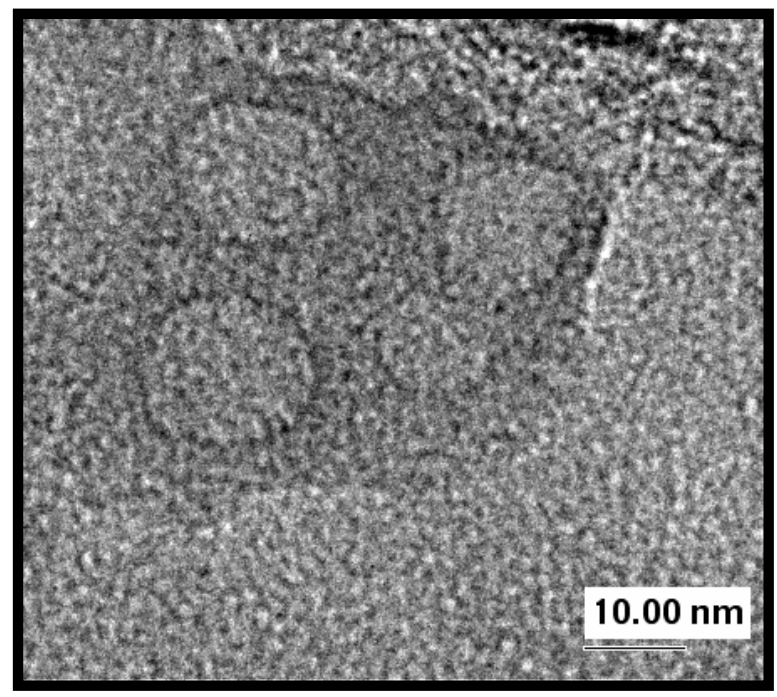

Figure S1. TEM image of ciprofloxacin@ $\mathrm{SiO}_{2}$ obtained by the $\mathrm{CN}$ - leaching method showing several particles. 


\section{Supporting information 3}

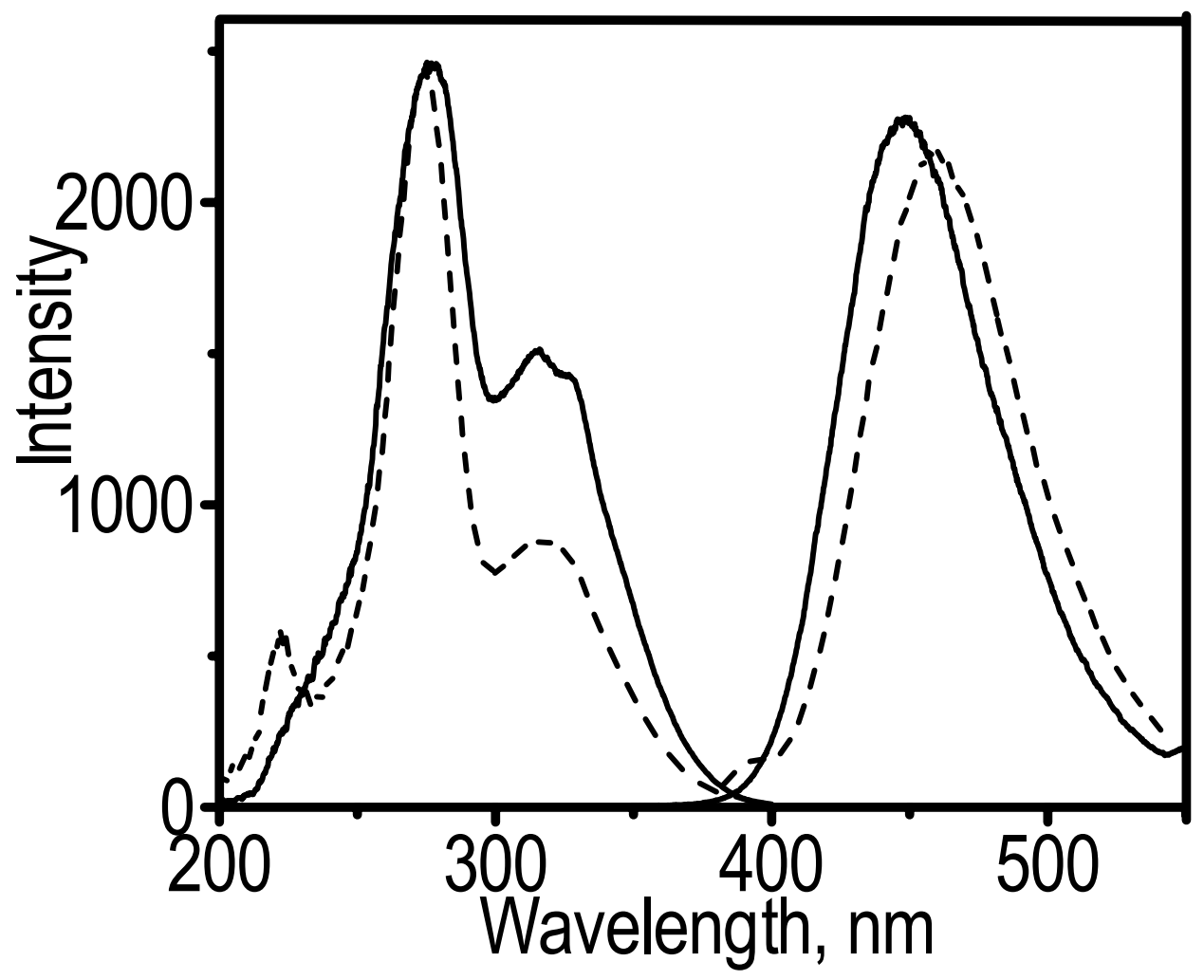

Figure S2. Emission and excitation spectra of free ciprofloxacin (solid line, $\lambda \max =448 \mathrm{~nm}$ ) and ciprofloxacin@SiO 2 (dotted line, $\lambda \max =458 \mathrm{~nm}$ ). For emission spectrum of ciprofloxacin@ $\mathrm{SiO}_{2}$, excitation was kept at $325 \mathrm{~nm}$. For the excitation spectrum, emission was kept at $458 \mathrm{~nm}$. 


\section{Supporting information 4}

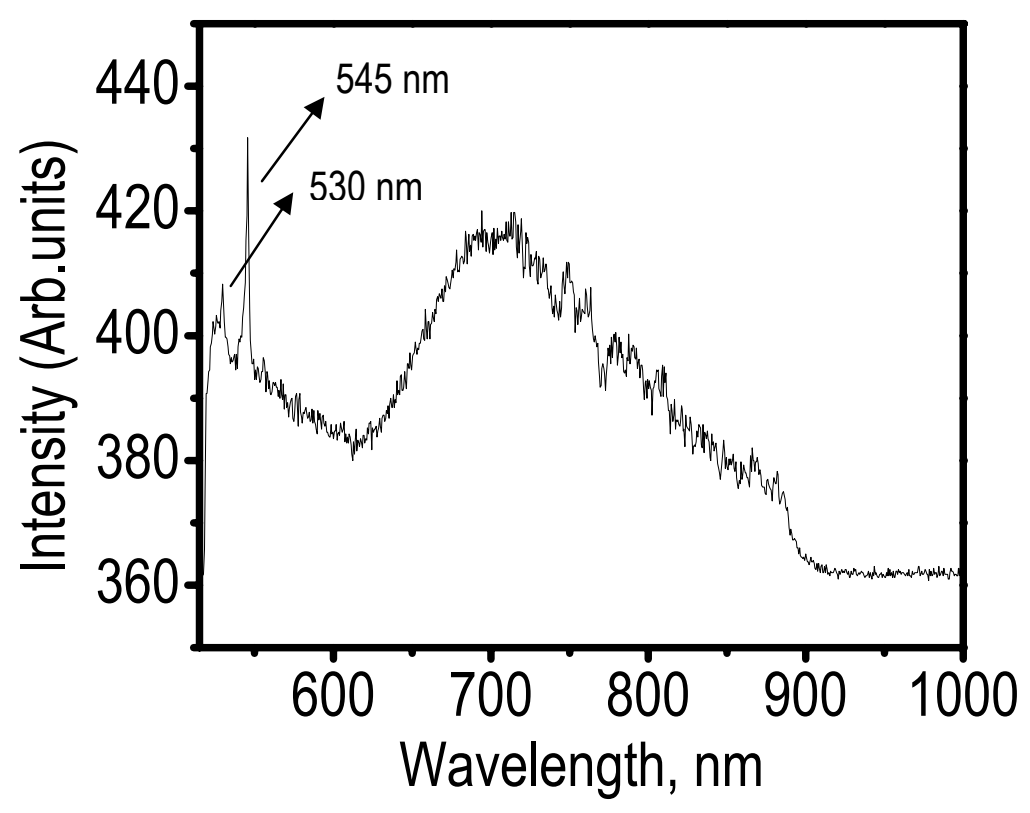

Figure S3. Fluorescence spectrum obtained after the incubation of FITC@SiO 2 with E. coli DH 5 $\alpha$ measured using WiTec Alpha-SNOM instrument with a $514.5 \mathrm{~nm}$ excitation laser. Apart from the broad $700 \mathrm{~nm}$ peak, there are two other features present in the $525 \mathrm{~nm}$ (Raman shift of $\sim 388 \mathrm{~cm}^{-1}$ ) region, which are the Raman features of the glass substrate used. The cut off after $900 \mathrm{~nm}$ is due to the limitation of the detector. 


\section{Supporting information 5}
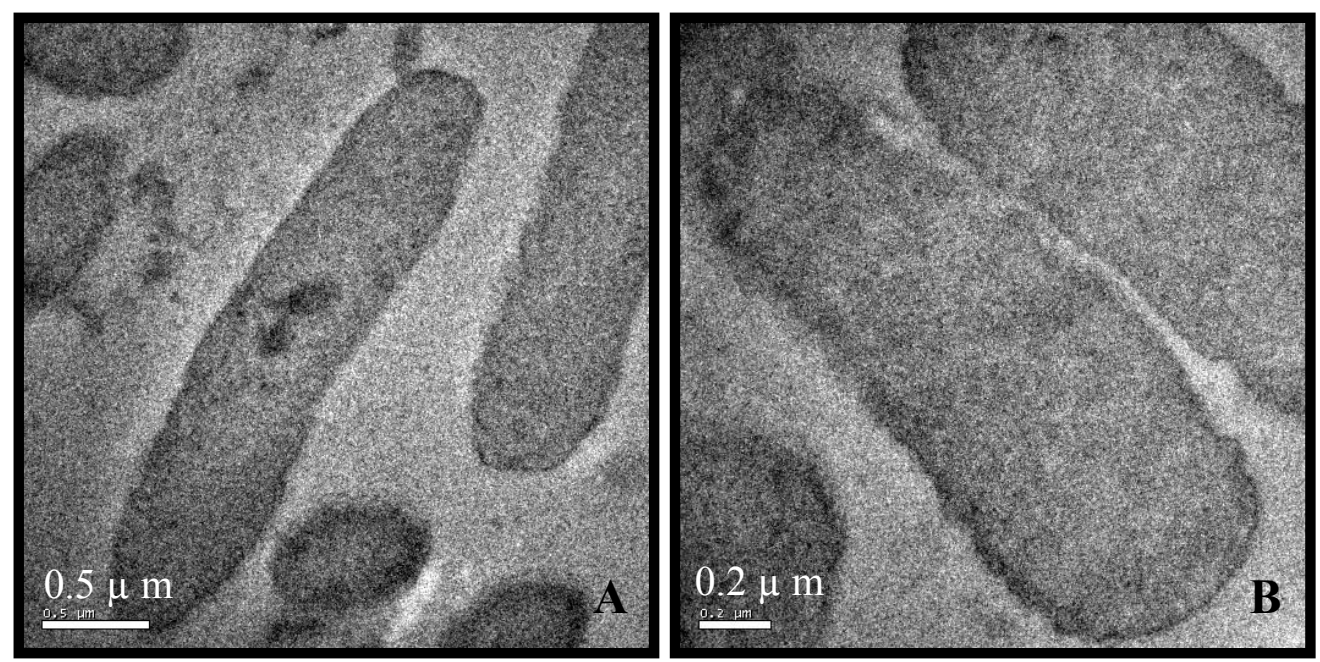

Figure S4. TEM images of free E. coli DH5 $\alpha$ (A and B). 